\title{
Refraction enhancement in plasmonics by coherent control of plasmon resonances
}

\author{
Panahpour, Ali; Mahmoodpoor, Abolfazl; Lavrinenko, Andrei V.
}

Published in:

Physical Review B

Link to article, DOI:

10.1103/PhysRevB.100.075427

Publication date:

2019

Document Version

Publisher's PDF, also known as Version of record

Link back to DTU Orbit

Citation (APA):

Panahpour, A., Mahmoodpoor, A., \& Lavrinenko, A. V. (2019). Refraction enhancement in plasmonics by coherent control of plasmon resonances. Physical Review B, 100(7), [075427 ].

https://doi.org/10.1103/PhysRevB.100.075427

\section{General rights}

Copyright and moral rights for the publications made accessible in the public portal are retained by the authors and/or other copyright owners and it is a condition of accessing publications that users recognise and abide by the legal requirements associated with these rights.

- Users may download and print one copy of any publication from the public portal for the purpose of private study or research.

- You may not further distribute the material or use it for any profit-making activity or commercial gain

- You may freely distribute the URL identifying the publication in the public portal

If you believe that this document breaches copyright please contact us providing details, and we will remove access to the work immediately and investigate your claim. 


\title{
Refraction enhancement in plasmonics by coherent control of plasmon resonances
}

\author{
Ali Panahpour, ${ }^{1}$ Abolfazl Mahmoodpoor, ${ }^{1}$ and Andrei V. Lavrinenko, ${ }^{2, *}$ \\ ${ }^{1}$ Javan Laser Company, Limited, 1464764511, Tehran, Iran \\ ${ }^{2}$ DTU Fotonik-Department of Photonics Engineering, Technical University of Denmark, Ørsteds pl. 343, DK-2800 Kongens Lyngby, Denmark
}

(Received 4 September 2018; revised manuscript received 29 March 2019; published 20 August 2019)

\begin{abstract}
Optical materials exhibit significant losses over the resonance frequency of their constituent atoms and so they are practically implemented at frequencies far from resonances. Electromagnetically induced transparency (EIT) provides a method for effective suppression of optical loss in a narrow window over the resonance, where the medium exhibits significant dispersion but at the expense of zero susceptibility. The classical or plasmonic analogs of the EIT effect are introduced and widely used in the context of electromagnetic or optical metamaterials (MMs). In another interesting phenomenon in quantum optics known as enhancement of index of refraction (EIR), the optical loss of the medium can be zero or even negative at the region of maximal susceptibility and negligible dispersion. This condition is interesting for applications where a strong electromagnetic response of the medium with negligible loss is required, such as zero- or negative-index metamaterials (MMs). Here we introduce a plasmonic analog of the EIR which allows for coherent control over the polarizability and absorption of plasmonic nanoantennas. It can open up the way for loss-compensated propagation of optical waves in zero-index to high-refractive-index plasmonic MMs. The scheme also offers an approach to all-optical switching and coherent control of transmission, diffraction, and polarization conversion properties of plasmonic nanostructures, as well as propagation properties of surface plasmon polaritons on metasurfaces.
\end{abstract}

DOI: 10.1103/PhysRevB.100.075427

The optical response of surface plasmons is mostly governed by metal and ambient medium parameters, geometry of structures, and also by plasmon hybridization, which can result in novel resonance line shapes, enabling the plasmonic systems to mimic some quantum optical effects such as Fano interference and electromagnetically induced transparency (EIT) [1-5]. The functionality of the plasmonic nanostructures is significantly improved through active plasmonics and specifically, by all-optical and ultrafast control of the surface plasmon on the nanoscale, which has been realized by exploiting the phase and polarization degrees of freedom of the exciting waves [6-10].

All-optical coherent control of surface plasmons in single or coupled nanoantennas is of fundamental importance, since they can be viewed as building blocks of plasmonic MMs, metasurfaces, and optical components such as sensors, switches, transistors, and light sources. Coherent control over the linear [6,10], nonlinear [11], and spatially nonlocal [12] responses of plasmonic nanoantennas has been reported, as well as controlling the directionality of emission, scattering patterns, and absorption in nanoparticles (NPs) involving nonlinear processes [13-15]. Moreover, coherent control of modal excitations in plasmonic metamolecules is investigated through adjusting the position of an exciting high-energy electron beam over a Dolmen-style resonator [16] or by changing the relative phase of two orthogonally polarized light fields, exciting a triple nanorod structure [17]. Nevertheless, controlling a resonant plasmonic nanoantenna to exhibit strong

*Corresponding author: alav@fotonic.dtu.dk polarizability and at the same time zero or negative absorption without involving materials with gain or nonlinear processes has not been reported so far.

Here, we propose a method for coherent control and manipulation of absorption and dispersion of a plasmonic nanoantenna using only the phase and polarization degrees of freedom of light. The approach is based on a classical analog of the quantum optics effect known as enhancement of the index of refraction (EIR), which occurs in atomic (or quantum dot) systems based on atomic coherence and quantum interference of different excitation pathways in variety of energy-level schemes [18-21].

To see how the usual resonant dispersion is altered in EIR and how it differs from EIT, the corresponding dispersion curves are shown in Fig. 1. The optical response of a two-level atomic system, represented by the complex susceptibility $\chi$, is typically of the form shown in Fig. 1(a). Here, refraction $[\operatorname{Re}(\chi)]$ is maximum at point $A$, where absorption $[\operatorname{Im}(\chi)]$ is also very large. In contrast, the EIT, usually occurring in threelevel systems, modifies the dispersion profile to the typical form shown in Fig. 1(b). Here, absorption is minimal at point B corresponding to highest dispersion and zero susceptibility. While EIT is ideal for slow light applications and enhancement of nonlinear interactions, it is not so promising when strong electromagnetic response (large linear susceptibility) is required. Such a strong response accompanied with vanishing absorption arises in EIR, where the dispersion is modified to the form shown in Fig. 1(c).

According to this figure, susceptibility (depicted as a function of the normalized probe detuning from the transition frequency of the excited level) is large at point $\mathrm{C}$, where 


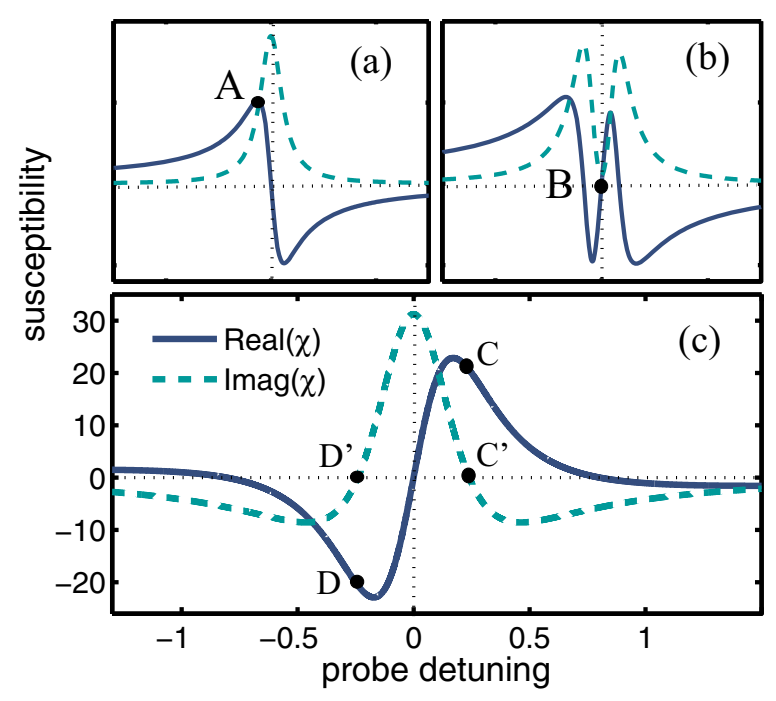

FIG. 1. Real and imaginary parts of susceptibility in an (a) twolevel system with standard dispersion curves, (b) coherent system showing EIT, and (c) coherent system with enhanced refraction through the EIR effect.

absorption totally vanishes (point $\mathrm{C}^{\prime}$ ). This results in huge lossless refractive indices in quantum coherent systems [21]. Additionally, susceptibility is negative at point $\mathrm{D}$, where absorption is again zero (point $\mathrm{D}^{\prime}$ ). This spectral region is interesting for development of lossless negative- or zeropermittivity $[\operatorname{Re}(\chi)=-1]$ media through proper tuning of the system parameters. Such implications are equally desirable in classical systems and can result in a variety of novel applications in optics and nanophotonics, and specifically in plasmonics, where conventional methods of loss compensation are still challenging [22-24].

Here, to introduce a simple toy model as a classical analog of the EIR we study a mass-spring system consisting of two linearly coupled one-dimensional oscillators (Fig. 2) with coupling constant $C$ and damping factors $\gamma_{1}$ and $\gamma_{2}$.

The complex amplitudes of the harmonic oscillators $x_{j}(t)=x_{j} \exp (-i \omega t)$ and driving forces $F_{j}(t)=$ $F_{j} \exp (-i \omega t)$ are connected through

$$
\left(\begin{array}{l}
x_{1} \\
x_{2}
\end{array}\right)=\left(\begin{array}{cc}
\delta_{1} & -C \\
-C & \delta_{2}
\end{array}\right)^{-1}\left(\begin{array}{l}
F_{1} / m \\
F_{2} / m
\end{array}\right)
$$

where $\delta_{j}=\omega_{0}^{2}-\omega^{2}-i \gamma_{j} \omega$ and $j=1$, 2. Assuming $F_{1}=$ $F_{01}, F_{2}=\beta F_{02}, x_{1}=x_{01}$, and $x_{2}=\beta x_{02}$, where $F_{01}$ and $F_{02}$ are real numbers and $\beta=\exp (-i \theta)$ is a complex parameter depending on the phase difference $\theta$ between the forces, we

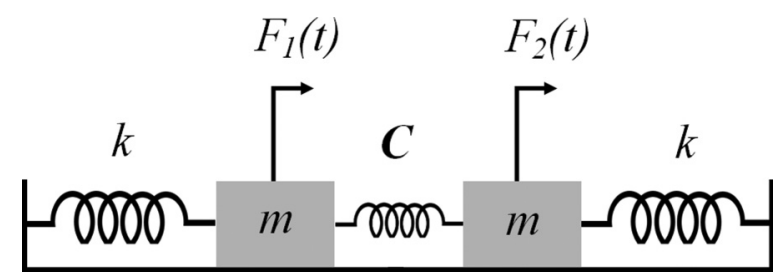

FIG. 2. Mass-spring analog of the EIR consisting of two linearly coupled one-dimensional oscillators.
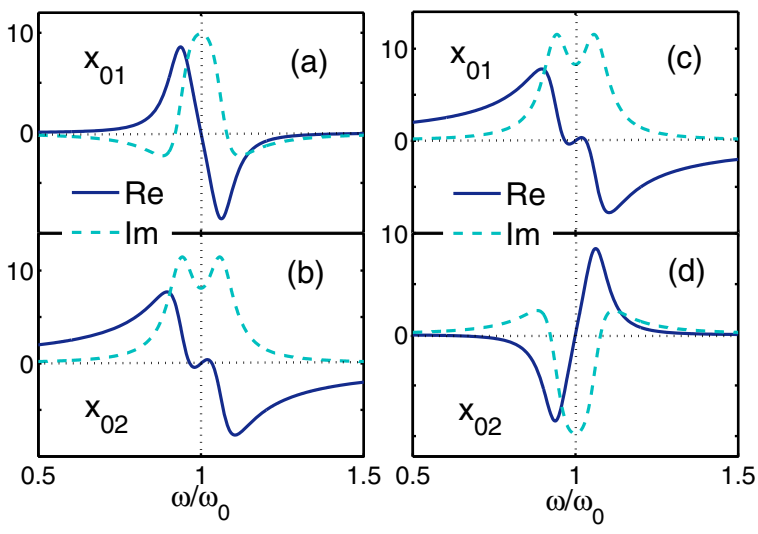

FIG. 3. Real and imaginary parts of $x_{01}$ and $x_{02}$ vs normalized frequency for $F_{01} \ll F_{02}$ (a), (b) and for $F_{01} \gg F_{02}$ (c), (d), correspondingly.

obtain (star sign denotes the complex conjugate)

$$
x_{01}=\frac{1}{m}\left(\frac{\delta_{2} F_{01}+\beta C F_{02}}{\delta_{1} \delta_{2}-C^{2}}\right), x_{02}=\frac{1}{m}\left(\frac{\delta_{1} F_{02}+\beta^{*} C F_{01}}{\delta_{1} \delta_{2}-C^{2}}\right),
$$

and time-averaged power absorbed by each oscillator is given by $W_{j}=\left\langle\operatorname{Re}\left[F_{j}(t)\right] \times \operatorname{Re}\left[\dot{x}_{j}(t)\right]\right\rangle_{t}=\frac{F_{0 j} \omega}{2} \operatorname{Im}\left(x_{0 j}\right)$.

The EIT effect can be reproduced in this system by adopting $\beta=0$, which means that only the first oscillator is coupled to the external force, and the second one is exclusively driven by virtue of coupling $C$. However, when both of the oscillators are driven by forces having some phase difference $\theta$, the system exhibits other interesting features. We assume the parameters $F_{01} / m \omega_{0}^{2}=0.01, F_{02} / m \omega_{0}^{2}=1, C / \omega_{0}^{2}=0.06$, $\gamma_{1}=\gamma_{2}=0.05 \omega_{0}$, and $\theta=\pi / 2$ in (2) and plot the real and imaginary parts of the complex amplitudes $x_{01}$ and $x_{02}$ in Figs. 3(a) and 3(b), respectively. By interchanging the values of the driving forces $\left(F_{01} / m \omega_{0}^{2}=1\right.$ and $\left.F_{02} / m \omega_{0}^{2}=0.01\right)$ and other parameters as before, the complex amplitudes $x_{01}$ and $x_{02}$ are changed to the forms in Figs. 3(c) and 3(d). Interestingly, the curves in Figs. 3(a) and 3(d) resemble the dispersion curves of a medium with enhanced index of refraction [Fig. 1(c)]. Similar results would be obtained if we used the phase difference $\theta=-\pi / 2$. The only difference would be the interchange of curves in Figs. 3(a) and 3(d).

The amplitude curves are not very sensitive to small deviations from $\theta= \pm \pi / 2$. However, for large deviations of the phase difference from $\theta= \pm \pi / 2$ and especially getting closer to $\theta=0$ or $\theta= \pm \pi$, the amplitude curves gradually convert to those of uncoupled oscillators.

According to Fig. 3, the complex amplitudes show negative absorption in specific spectral ranges around the resonance. However, using relations (2) it can be shown that the total power absorbed by both oscillators together $W=(\omega / 2)\left[F_{01} \operatorname{Im}\left(x_{01}\right)+F_{02} \operatorname{Im}\left(x_{02}\right)\right]$ is positive at all frequencies.

The complex amplitude curves in Figs. 3(a) and 3(d) inspire the application of microscopic coupled dipole oscillators with similar responses as the constitutive elements of 


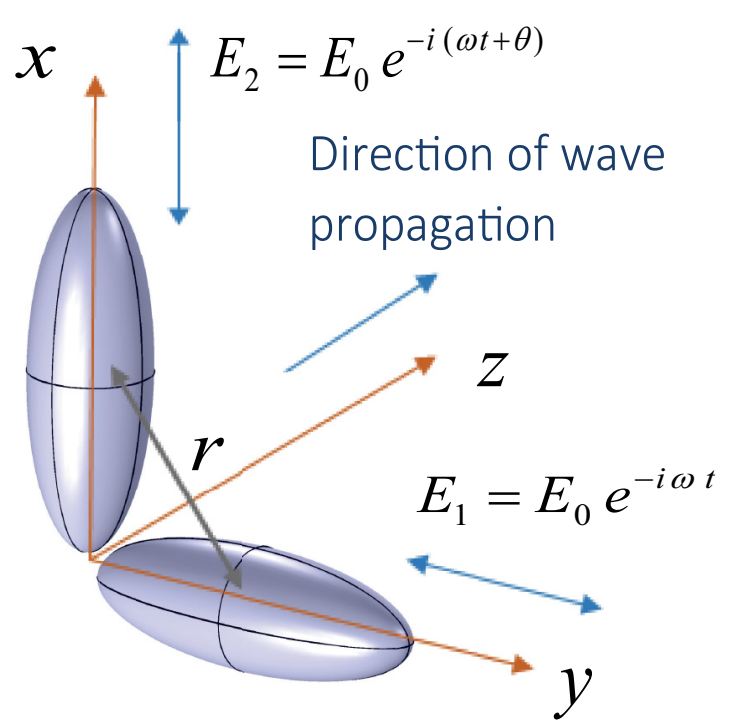

FIG. 4. A plasmonic molecule consisting of two perpendicular silver spheroidal nanoparticles excited by two orthogonal polarizations having a phase difference $\theta$.

a medium demonstrating the classical EIR. Metallic coupled nanoantennas as subwavelength constituent elements of plasmonic MMs can be an interesting platform, because the zero-absorption feature in the EIR phenomenon can be exploited as a loss compensation mechanism in notoriously lossy plasmonic nanostructures.

To this end, we consider a specific metamolecule consisting of two coupled plasmonic nanoantennas which can be metal strips or nanorods. To simplify the analytical calculations, we consider two perpendicular prolate (cigar-shaped) spheroidal NPs shown in Fig. 4. They are excited by two plane waves with electric fields $E_{1}=E_{01} e^{-i \omega t}$ and $E_{2}=$ $E_{02} e^{-i(\omega t+\theta)}$, having perpendicular polarizations in the $x$ and $y$ directions, and frequency $\omega$ close to the localized surface plasmon resonance of the NPs along their longer semiaxes. The direction of propagation of the $x(y)$-polarized wave can be in any direction in a plane orthogonal to the $x(y)$ axis. For $E_{01} \ll E_{02}$ or $E_{01} \gg E_{02}$, and $\theta=\pi / 2$, the curves for complex polarizability of the NPs are quite similar to the plots in Fig. 3, corresponding to the conditions of $F_{01} \ll F_{02}$ and $F_{01} \gg F_{02}$. To study EIR in the condition of comparable pump and probe fields, we assume exciting fields with equal amplitudes. By taking $\theta=\pi / 2$, the exciting fields with the same direction of propagation (Fig. 4) are actually the components of a circularly polarized wave.

The polarizability of a spheroidal NP of volume $v$ in the electrostatic limit is given by $\alpha=v\left(\varepsilon-\varepsilon_{h}\right) /\left[\varepsilon_{h}+L\left(\varepsilon-\varepsilon_{h}\right)\right]$ [25], where $\varepsilon$ and $\varepsilon_{h}$ are dielectric functions of the NP and host medium, respectively, and $L=\frac{1-e^{2}}{e^{2}}\left(-1+\frac{1}{2 e} \ln \frac{1+e}{1-e}\right)$ is a geometrical factor in terms of eccentricity $\left(e^{2}=1-b^{2} / a^{2}\right)$ of the NPs [25]. We assume the nanoantennas to be silver NPs in vacuum $\left(\varepsilon_{h}=1\right)$ with the dielectric function of Johnson and Christy [26]. The size and separation of the NPs are assumed much smaller than the wavelength so that they can be approximated as point dipoles with electrostatic approximation for their electric field.

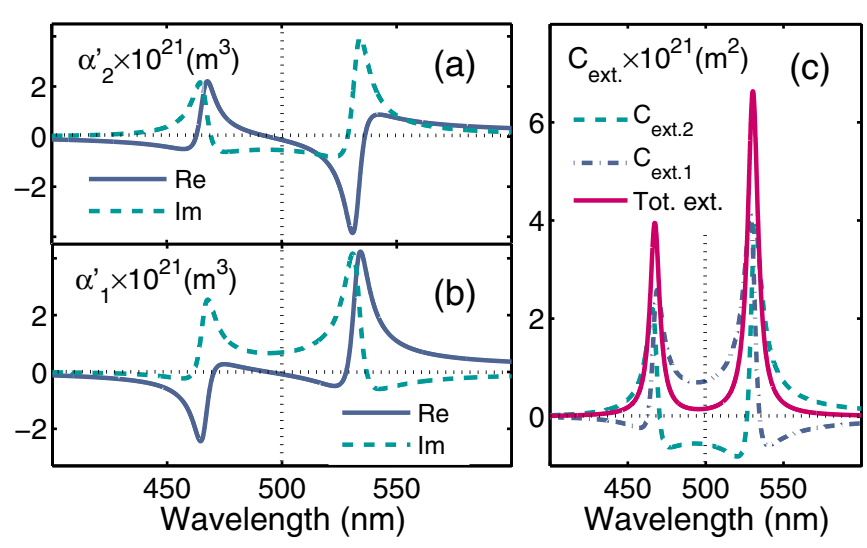

FIG. 5. Real and imaginary components of the polarizabilities (a) $\alpha_{2}^{\prime}$ and (b) $\alpha_{1}^{\prime}$ (of the coupled NPs in Fig. 4) as functions of wavelength, and (c) the individual and total ECSs of the same NPs.

The induced dipole moment along the longer semiaxis of each NP is affected by the superposition of the incident electric field and field induced by the dipole moment of the other NP. The projection of the latter on the longer semiaxis is proportional to dipole moment $\mathbf{p}$ with coefficient $C$ depending on the NP's separation and its orientation. Therefore, the polarizabilities of the coupled NPs (in terms of $\alpha$ and $\beta=$ $e^{-i \theta}$ ) defined by the relations $p_{1}=\varepsilon_{0} \alpha_{1}^{\prime} E_{0}$ and $p_{2}=\varepsilon_{0} \alpha_{2}^{\prime} \beta E_{0}$ are obtained as $\alpha_{1}^{\prime}=\alpha \frac{1+\beta C \alpha}{1-C^{2} \alpha^{2}}$ and $\alpha_{2}^{\prime}=\alpha \frac{1+\beta^{*} C \alpha}{1-C^{2} \alpha^{2}}$.

Using the expression for the extinction cross section (ECS) of a dipole, excited by an incident plane wave $C_{\text {ext. }}=$ $\left(k / \varepsilon_{0}\left|E_{0}\right|^{2}\right) \operatorname{Im}\left(\mathbf{E}_{\text {inc }}^{*} \cdot \mathbf{p}\right)$ [27], the extinction of the linearly polarized components of the incident wave by the corresponding NPs can be calculated from $C_{\text {ext. } j}=k \operatorname{Im}\left(\alpha^{\prime}{ }_{j}\right),(j=1,2)$. We consider spheroidal NPs with the semiaxis dimensions $b=c=10 \mathrm{~nm}$ and $a=30 \mathrm{~nm}$ in the configuration of Fig. 4 . The real and imaginary components of the polarizabilities $\alpha_{1}^{\prime}$ and $\alpha_{2}^{\prime}$ (along the longer semiaxis of the NPs) as functions of wavelength for parameters $r=50 \mathrm{~nm}$ and $\theta=\pi / 2$ are plotted in Fig. 5.

We see that in the case of equal amplitude exciting fields, both of the NPs show negative extinction in different spectral ranges and exhibit zero absorption near the positive and negative peak values of their real polarizability. The individual and total ECSs of the NPs are represented in Fig. 5(c). The ECS of each NP is negative in specific spectral ranges, but the total ECS is positive at all wavelengths as expected.

The polarizability values in Figs. 5(a) and 5(b) show that a three-dimensional (3D) array of such metamolecules with the unit-cell dimension of $100 \mathrm{~nm}$ (density $10^{21} \mathrm{~m}^{-3}$ ) can exhibit zero or negative permittivity at the lossless portion of the spectrum.

To support the analytical results, interaction of the incident circularly polarized wave with the NPs in Fig. 4 is simulated in the frequency domain based on the finite-element method. The computational region is limited to the interior region of a perfect electric conductor (PEC) cylinder of diameter $D=400 \mathrm{~nm}$ as a circular waveguide with input and output ports. The circularly polarized wave is approximated by a combination of two orthogonal $\mathrm{TE}_{11}$ modes with a $\pi / 2$ phase difference. The waveguide contains the coupled spheroidal 


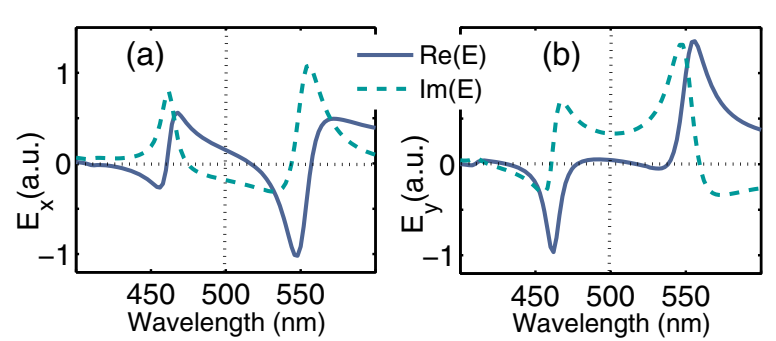

FIG. 6. Real and imaginary parts of (a) $x$ and (b) $y$ components of the electric field near the NPs oriented in the respective $x$ and $y$ directions when the $x$-polarized field precedes the $y$-polarized field by $\theta=\pi / 2$ inside the waveguide.

NPs with the same geometrical dimensions as before and with the dielectric function model of Johnson and Christy [26]. The real and imaginary parts of the $x(y)$ component of the electric field near the NP oriented in $x(y)$ direction excited by the mode preceded (delayed) by $\theta=\pi / 2$ in the waveguide is plotted in Fig. 6(a) [6(b)]. The electric field is a sum of the contributions from the incident field and the dipole response of the coupled NPs. Therefore, the field near each NP can give insight into the effective polarizability of the particle.

The curves in Fig. 6 are quite similar to those in Figs. 5(a) and 5(b), except for the spectral separations between the resonances, which are closer to each other in Fig. 5. The discrepancies between the simulation and analytical results can be attributed to the introduction of higher-order (beyond the dipolar) interactions due to the close proximity of the NPs in simulation, which also results in stronger coupling of the NPs compared to the conditions of analytical calculations where the NPs are approximated by point dipoles.

The canalization of energy from one polarization to another through coupled NPs can be illustrated by tracing the streamlines of the power flow from the input to output ports of the circular waveguide. Figure 7(a) [7(b)] shows the power flow streamlines around the NPs, corresponding to a wavelength of $\lambda=500 \mathrm{~nm}(\lambda=440 \mathrm{~nm})$, at which there is canalization of energy from $y(x)$ to $x(y)$-polarized field components through mutual coupling of the NPs.

As expected, according to Fig. 7(a), the majority of the streamlines are headed for the horizontal absorptive NP and then redirected toward the vertical NP and finally steered toward the output port. Figure 7(c) shows a closer view of the NPs, with a small number of streamlines to be traced with more clarity. Similarly, in Figs. 7(b) and 7(d) (corresponding to $\lambda=440 \mathrm{~nm}$ ) streamlines are mainly directed to the vertical NP with positive extinction and then redirected toward the output port by the horizontal NP having negative extinction. This property of power exchange between normal polarizations may find applications in plasmonic polarization conversion metasurfaces [28,29], with an augmented functionality of all-optical control over the conversion efficiency.

This mode conversion or energy exchange between the orthogonal polarizations can be verified by the time-domain finite-element method. Simulation of the mode conversion in time domain is challenging in a circular waveguide, because simultaneous extinction of one polarization and growth of the other cannot be distinguished at the output port. Therefore

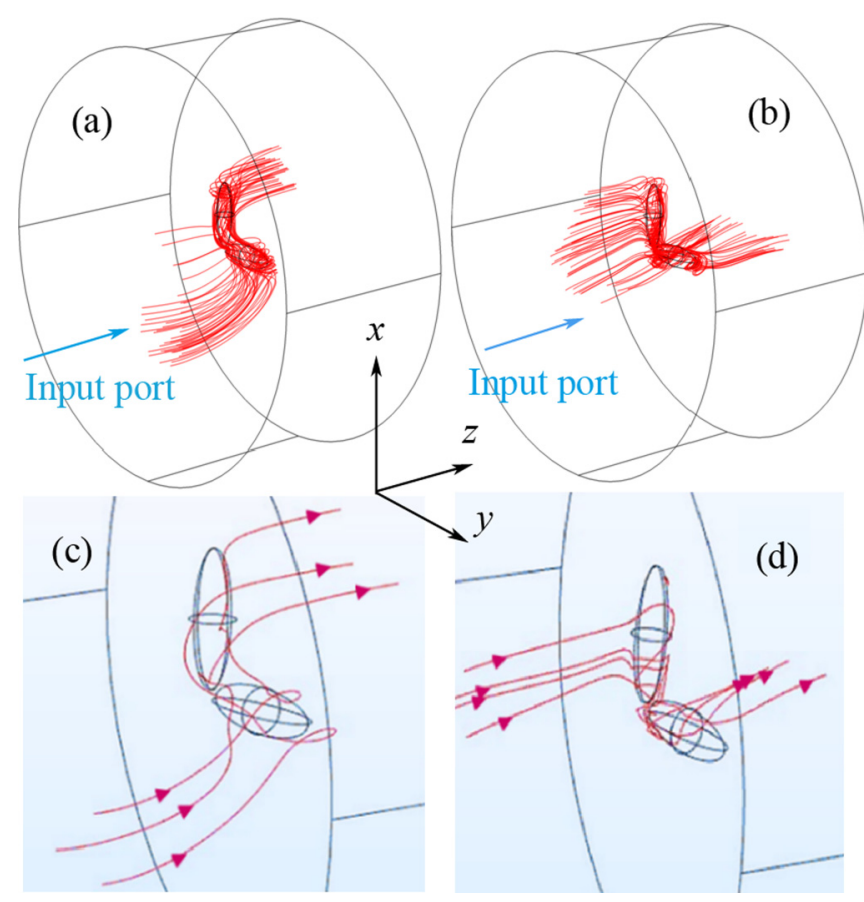

FIG. 7. Power flow streamlines around the NPs in the circular waveguide with the same parameters and geometrical dimensions as before and at wavelengths (a, c) $\lambda=500 \mathrm{~nm}$ and (b, d) $\lambda=440 \mathrm{~nm}$.

we use a configuration depicted in Fig. 8, where there are two separate waveguides with orthogonal electric fields corresponding to each NP.

The waveguides consist of two pairs of perfect electric and perfect magnetic conductor (PMC) sidewalls as well as input

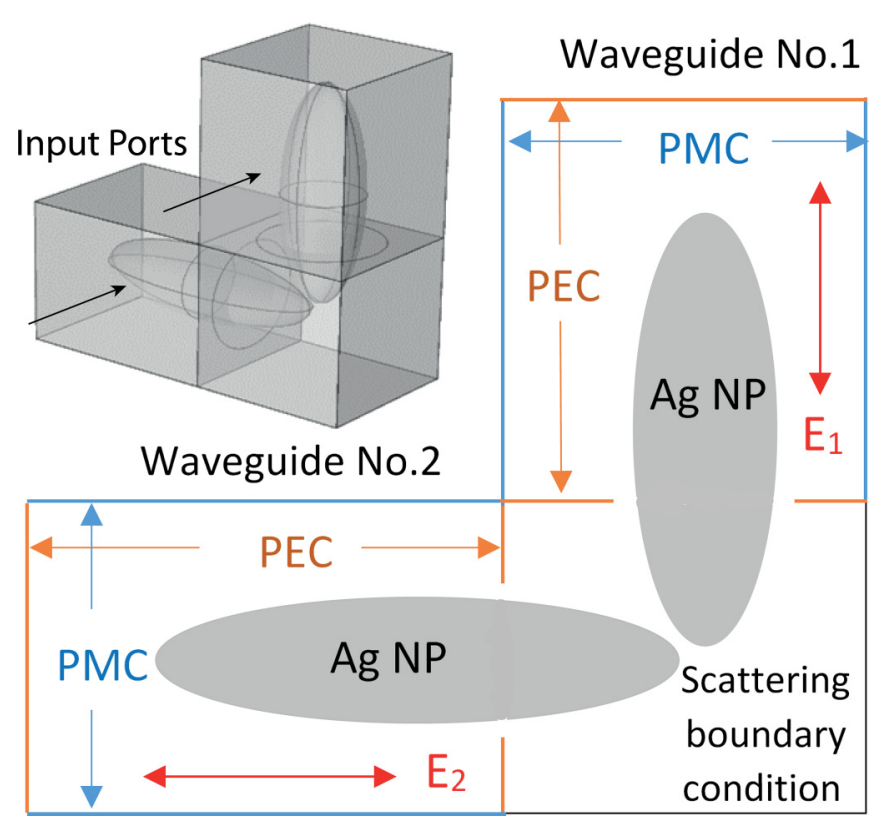

FIG. 8. Two waveguides with orthogonal electric fields corresponding to each NP of the metamolecule. The waveguides have two perfect electric and two perfect magnetic conductor sidewalls as well as input ports shown in the $3 \mathrm{D}$ picture with output ports in front of them. 


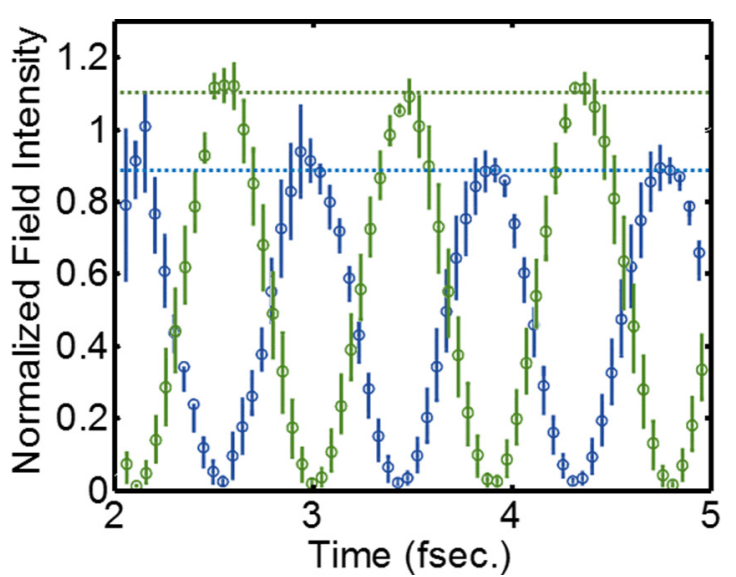

FIG. 9. Normalized field intensity at the output ports of waveguides no. 1 (green curve) and no. 2 (blue curve) in Fig. 8.

and output electromagnetic ports. The mutual coupling of the NPs is enabled by two small holes in the waveguide walls. Electromagnetic fields with equal amplitudes and $\theta=\pi / 2$ phase difference are fed into the waveguides from the input ports, and the field intensity is calculated as a function of time at the output ports. Field $E_{1}$ fed into waveguide no. 1 is preceding field $E_{2}$ in the other waveguide by $\theta=\pi / 2$. Simulations with the NP parameters and dimensions as before $(\lambda=500 \mathrm{~nm})$ show (Fig. 9) that the output field intensities deviated from those in the case $\theta=0$ with nearly $10 \%$ growth for waveguide no.1 and nearly $10 \%$ attenuation for no.2, revealing the power transfer between waveguides.

The circles in Fig. 9 correspond to the calculations at different time steps, and the bars represent the calculation errors due to the size of the mesh elements.

In summary, we introduced a classical mass-spring analog and a plasmonic counterpart of the quantum optics effect of enhancement of index of refraction. The analogy shows that maximal polarizability of a plasmonic nanoantenna can be achieved accompanied by zero absorption of the probe field. Therefore, it offers the way for development of losscompensated plasmonic MMs and metasurfaces with tunable permittivities ranging from zero to maximal values. The classical EIR does not involve any gain or nonlinear materials and is based totally on polarizations and phases of electromagnetic waves. It can also find applications in all-optical switching or optically tunable polarization conversion metasurfaces.

A.V.L. acknowledges partial support from the Villum Fonden "DarkSILD" project (No. 11116).
[1] B. Luk'yanchuk, N. I. Zheludev, S. A. Maier, N. J. Halas, P. Nordlander, H. Giessen, and C. T. Chong, The Fano resonance in plasmonic nanostructures and metamaterials, Nat. Mater. 9, 707 (2010).

[2] S. Zhang, Dentcho A. Genov, Y. Wang, M. Liu, and X. Zhang, Plasmon-Induced Transparency in Metamaterials, Phys. Rev. Lett. 101, 047401 (2008).

[3] N. Papasimakis, V. A. Fedotov, N. I. Zheludev, and S. L. Prosvirnin, Metamaterial Analog of Electromagnetically Induced Transparency, Phys. Rev. Lett. 101, 253903 (2008).

[4] N. Liu, L. Langguth, T. Weiss, J. Kästel, M. Fleischhauer, T. Pfau, and H. Giessen, Plasmonic analogue of electromagnetically induced transparency at the Drude damping limit, Nat. Mater. 8, 758 (2009).

[5] P. Tassin, L. Zhang, R. Zhao, A. Jain, T. Koschny, and C. M. Soukoulis, Electromagnetically Induced Transparency and Absorption in Metamaterials: The Radiating Two-Oscillator Model and Its Experimental Confirmation, Phys. Rev. Lett. 109, 187401 (2012).

[6] M. Durach, A. Rusina, M. I. Stockman, and K. Nelson, Toward full spatiotemporal control on the nanoscale, Nano Lett. 7, 3145 (2007).

[7] M. I. Stockman, Ultrafast nanoplasmonics under coherent control, New J. Phys. 10, 025031 (2008).

[8] M. Aeschlimann, M. Bauer, D. Bayer, T. Brixner, F. J. García De Abajo, W. Pfeiffer, M. Rohmer, C. Spindler, and F. Steeb, Adaptive subwavelength control of nano-optical fields, Nature (London) 446, 301 (2007).

[9] J.-S. Huang, D. V. Voronine, P. Tuchscherer, T. Brixner, and B. Hecht, Deterministic spatiotemporal control of optical fields in nanoantennas and plasmonic circuits, Phys. Rev. B 79, 195441 (2009).
[10] M. Sukharev and T. Seideman, Phase and polarization control as a route to plasmonic nanodevices, Nano Lett. 6, 715 (2006).

[11] M. Abb, P. Albella, J. Aizpurua, and O. L. Muskens, All-optical control of a single plasmonic nanoantenna-ITO hybrid, Nano Lett. 11, 2457 (2011).

[12] D. McArthur, B. Hourahine, and F. Papoff, Coherent control of plasmons in nanoparticles with nonlocal response, Opt. Commun. 382, 258 (2017).

[13] S. Roke, M. Bonn, and A. V. Petukhov, Nonlinear optical scattering: The concept of effective susceptibility, Phys. Rev. B 70, 115106 (2004).

[14] S. G. Rodrigo, H. Harutyunyan, and L. Novotny, Coherent Control of Light Scattering from Nanostructured Materials by Second-Harmonic Generation, Phys. Rev. Lett. 110, 177405 (2013).

[15] F. Papoff, D. McArthur, and B. Hourahine, Coherent control of radiation patterns of nonlinear multiphoton processes in nanoparticles, Sci. Rep. 5, 12040 (2015).

[16] T. Coenen, D. T. Schoen, S. A. Mann, S. R. K. Rodriguez, B. J. M. Brenny, A. Polman, and M. L. Brongersma, Nanoscale spatial coherent control over the modal excitation of a coupled plasmonic resonator system, Nano Lett. 15, 7666 (2015).

[17] F. Zeuner, M. Muldarisnur, A. Hildebrandt, J. Förstner, and T. Zentgraf, Coupling mediated coherent control of localized surface plasmon polaritons, Nano Lett. 15, 4189 (2015).

[18] M. O. Scully, Enhancement of the Index of Refraction via Quantum Coherence, Phys. Rev. Lett. 67, 1855 (1991).

[19] M. O. Scully, From lasers and masers to phaseonium and phasers, Phys. Rep. 219, 191 (1992).

[20] M. Fleischhauer, C. H. Keitel, M. O. Scully, C. Su, B. T. Ulrich, and S.-Y. Zhu, Resonantly enhanced refractive index without absorption via atomic coherence, Phys. Rev. A 46, 1468 (1992). 
[21] H. F. Zhang, J. H. Wu, X. M. Su, and J. Y. Gao, Quantuminterference effects on the index of refraction in $\mathrm{anEr}^{3+}$-doped yttrium aluminum garnet crystal, Phys. Rev. A 66, 053816 (2002).

[22] V. E. Babicheva, I. V. Kulkova, R. Malureanu, K. Yvind, and A. V. Lavrinenko, Plasmonic modulator based on gain-assisted metal-semiconductor-metal waveguide, Photonics Nanostruct. Fundam. Appl. 10, 389 (2012).

[23] V. V. Klimov, I. V. Zabkov, D. V. Guzatov, and A. P. Vinogradov, Loss compensation symmetry in dimers made of gain and lossy nanoparticles, Laser Phys. Lett. 15, 035901 (2018).

[24] A. R. Rashed, A. De Luca, R. Dhama, A. Hosseinzadeh, M. Infusino, M. El Kabbash, S. Ravaine, R. Bartolino, and G. Strangi, Battling absorptive losses by plasmon-exciton coupling in multimeric nanostructures, RSC Adv. 5, 53245 (2015).
[25] C. F. Bohren and D. R. Huffman, Absorption and Scattering of Light by Small Particles, Wiley Professional Paperback Edition (Wiley, New York, 1998).

[26] P. B. Johnson and R. W. Christy, Optical constants of the noble metals, Phys. Rev. B 6, 4370 (1972).

[27] S. Zou, N. Janel, and G. C. Schatz, Silver nanoparticle array structures that produce remarkably narrow plasmon line shapes, J. Chem. Phys. 120, 10871 (2004).

[28] Z. Li, W. Liu, H. Cheng, S. Chen, and J. Tian, Realizing broadband and invertible linear-to-circular polarization converter with ultrathin single-layer metasurface, Sci. Rep. 5, 18106 (2015).

[29] E. O. Owiti, H. Yang, C. F. Ominde, and X. Sun, Broadband quarter-wave plate based on dielectricembedded plasmonic metasurface, RSC Adv. 7, 37495 (2017). 